\title{
Predappio: un luogo della memoria fascista
}

\author{
di Elena Gnagnetti
}

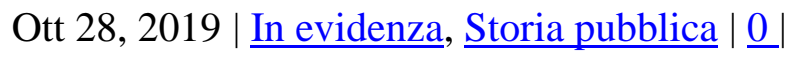

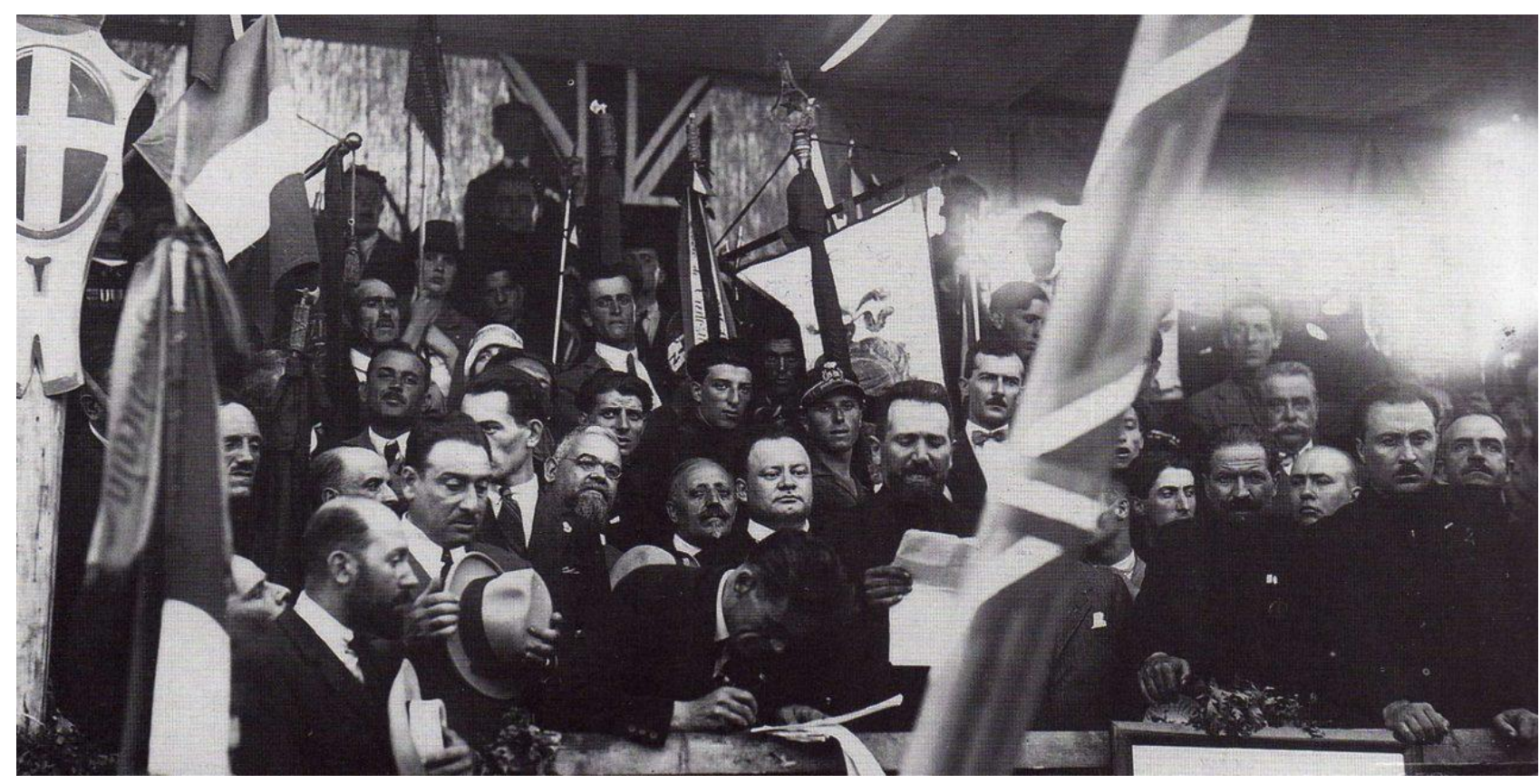

Cerimonia ufficiale per la fondazione di Predappio Nuova - Autore sconosciuto $\underline{\text { Pubblico dominio, }}$ Collegamento

\begin{abstract}
L'articolo ripercorre sinteticamente le tappe che hanno caratterizzato l'ideazione a Predappio del progetto museografico dedicato alla storia del fascismo e il dibattito pubblico che ne è conseguito. La scelta del luogo ha sollevato molte perplessità a causa del suo potere fortemente evocativo. Predappio è da sempre meta di pellegrinaggi per coloro che vogliono celebrare il duce. La persistenza del suo ricordo nell'immaginario collettivo è dovuta non solo alla presenza della tomba e dei negozi che vendono souvenir fascisti, ma è da ricercarsi anche nel mancato avvio di una visione autocritica del passato e di una riflessione più attenta sulle modalità di ricostruzione e trasmissione della storia.

Negli ultimi anni l'indagine storiografica si è molto concentrata sulla ricerca e sulla mappatura dei luoghi che hanno lasciato nel territorio una traccia materiale o ideale di alcuni eventi del passato. Ricordare attraverso il luogo significa, infatti, non solo recuperare lo spazio fisico di alcuni accadimenti della storia locale, ma anche individuare e interpretare le sue stratificazioni d'uso, le modalità con cui le comunità vi interagiscono o vi si rispecchiano nel presente. «La memoria è il regno della storia sociale»[1], ha osservato Mario Isnenghi, perché in essa si conserva l'identità del gruppo, le sue abitudini, le sue convenzioni e le sue esperienze. In questa prospettiva la mappatura dei luoghi della memoria non è solo, la localizzazione spaziale degli eventi, ma anche la ricostruzione geografica di un immaginario collettivo. Rivisitare i luoghi senza la consapevolezza della loro forza rappresentativa vuol dire trascurare una parte della loro essenza e avviare un intervento di ricostruzione storica approssimativo e deviante.
\end{abstract}


Tale premessa contribuisce a meglio chiarire alcune delle ragioni possibili che possono avere spinto, ormai qualche anno fa, l'ex sindaco di Predappio, Giorgio Frassineti, a promuovere la realizzazione di un centro di documentazione dedicato alla storia del fascismo a Predappio, città natale e luogo di sepoltura della salma di Benito Mussolini. Attraverso una ricostruzione rigorosa delle vicende che hanno caratterizzato il ventennio fascista l'iniziativa intende neutralizzare la sacralità del luogo e promuovere il superamento degli stereotipi mitologici generati dal fascismo. Si tratta di una necessità ampiamente condivisa, e tuttavia la scelta di realizzare quel progetto a proprio a Predappio ha sollevato un acceso dibattito pubblico. Alcuni intellettuali hanno espresso dubbi e scetticismo, e hanno espresso il timore che un'iniziativa simile realizzata in un territorio fortemente connotato dalla memoria nostalgica del fascismo potesse contribuire a enfatizzare il dato celebrativo.

L'assenza di un consenso unanime all'iniziativa dimostra la persistente presenza in Italia di una memoria ancora non condivisa e legata a dinamiche commemorative che minimizzano la storia e invalidano la conoscenza.

Ripercorrere, seppur sinteticamente, le fasi che hanno accompagnato l'ideazione del progetto e i punti essenziali del dibattito pubblico, rappresenta un'occasione significativa per prendere coscienza e riflettere sulle attuali modalità di ricostruzione e trasmissione della storia.

\section{Alcune considerazioni preliminari/Per capire cosa succede a Predappio}

Con l'ascesa di Benito Mussolini e il consolidarsi del regime fascista Predappio, città natale del duce, abbandona il suo status di piccolo e tranquillo borgo medievale per trasformarsi in un luogo di culto. Come testimonia Elisa Giovannetti, l'affluenza dei visitatori durante il ventennio fascista è così massiccia da richiedere l'istituzione di un ufficio propagandistico in grado di accogliere e gestire i turisti:

«Il fenomeno delle visite alla città natale era talmente esteso, in epoca fascista, da indurre Achille Starace, segretario del Partito nazionale fascista, a creare a Predappio un Ufficio propaganda, che fu istituito nel 1940 con sede nella Casa del Fascio. La sua attività ampliava le ordinarie funzioni di un ufficio turistico: oltre all'organizzazione delle visite, infatti, offriva servizi foto grafici di documentazione, oggetti-ricordo e cartoline con tanto di timbro, oltre alla ristorazione presso la stessa Casa del fascio.»[2]

Nel dopoguerra la città non perde il suo potere attrattivo. Nel periodo in cui la salma di Mussolini è tenuta nascosta nel convento di Cerro Maggiore (tra il 1946 e il 1957), a Predappio il ricordo del duce resta vivo. Significativa è la testimonianza di Vittore Querel pubblicata nel 1954 e che Massimo Baioni ricorda nel suo saggio dedicato a Predappio:

«Un'aria di tragedia e di sconforto domina questa Predappio piuttosto abbandonata come se le dovessero rimproverare una indicibile colpa. [...] L'atmosfera di Predappio è talmente impregnata di "Lui", del suo ricordo, del suo volto, delle sue parole e dei suoi gesti che non è facile pensare che vi possa essere un po' di posto libero per un'altra persona. [...] Ormai Predappio si porterà per generazioni il nome ed il ricordo di "Lui"».[3]

Secondo Querel tutta la città è fortemente segnata dal ricordo del duce che occupa idealmente l'intero spazio disponibile. E non solo. L'autore descrive, con tono devozionale, lo stato di 
abbandono della città equiparandolo ad un atto di profanazione. In quanto luogo di culto, Predappio impone un rispetto reverenziale che, tuttavia, è venuto meno dopo la caduta del regime.

Quando il 31 agosto 1957 la salma di Mussolini è collocata nella cripta di famiglia nel cimitero di San Cassiano di Predappio qualcosa cambia. Da quel momento la tomba del duce diventa il sacrario per eccellenza della pratica votiva neofascista, uno strumento fondamentale per preservare il ricordo del duce.

È un processo niente affatto inusuale; come ricorda lo storico dell'arte Hans Belting, la capacità di perdurare è nella natura ambivalente dell'immagine tombale:

"L'immagine di un defunto, dunque, non è un'anomalia ma addirittura il senso originario di ciò che comunque è un 'immagine. Il morto è sempre un assente, la morte un'assenza intollerabile che si vorrebbe colmare per mezzo di un'immagine. Per questo gli uomini hanno collocato i loro morti in un luogo ben determinato (la tomba) e dato loro, attraverso l'immagine, un corpo immortale; un corpo simbolico con il quale essere risocializzati mentre il corpo mortale si dissolve.»[4]

La tomba di Mussolini garantisce, dunque, la sopravvivenza nella memoria collettiva di ciò che in natura è destinato a dissolversi, è il luogo sociale che attiva un'azione, cioè un atto di animazione condiviso che dona vitalità all'immagine di Mussolini.

Alle pratiche commemorative si aggiunge anche la vendita di cimeli fascisti in vari negozi della cittadina. Si tratta di oggetti, solo in apparenza inanimati, ma che hanno il potere di innescare in chi li acquista una reazione idolatrante. Lo storico dell'arte David Freedberg li identifica come elementi costitutivi dei pellegrinaggi:

«Tali immagini finiscono allora col diventare dei memento surrettizi, ma insistenti, di poteri e meriti che trascendono di molto il misero rispetto che accordiamo loro, e poi d'improvviso, senza farsi notare, come per un atto furtivo, ecco che sono dei feticci e diventano talismani.»[5]

Questi oggetti-copia dell'originale ne assorbono il potere e ne mantengono vivo il ricordo. Sono un feticcio, un amuleto da conservare e venerare: incarnazione vivente di ciò che rappresentano.

Come accade a chi si reca in pellegrinaggio in un luogo di culto, così anche a Predappio il visitatore si aspetta di vivere un'esperienza spirituale: nostalgici e neofascisti visitano la città, commemorano la tomba e acquistano souvenir, perché sono convinti che queste pratiche garantiscano l'incontro con il duce. In questo senso si può dire che la visita a Predappio sia solo l'atto finale di un processo di condizionamento e indottrinamento iniziato prima e altrove.

\section{Breve sintesi delle tappe del progetto dell'Ex casa del fascio e dell'ospitalità di Predappio}

L'idea di recuperare l'Ex casa del fascio e dell'ospitalità di Predappio nasce alcuni anni fa e precisamente nel febbraio 2014 quando Carlo Giunchi presenta il Programma di riutilizzo e gestione, commissionato al Comune di Predappio dall'Unione dei comuni della Romagna forlivese. $\mathrm{Nel}$ documento sono descritti lo stato di conservazione dell'edificio e gli interventi di recupero da attuare per ospitare un progetto di valorizzazione culturale. Nel 2015 il comitato consultivo, nominato dal Comune di Predappio e coordinato da Marcello Flores, presenta le Linee guida per il progetto del museo storico previsto nell'ambito del riuso dell'Ex casa del fascio e dell'ospitalità di Predappio. L'obiettivo esplicitato è quello di valorizzare la memoria storica della città attraverso 
una ricostruzione critica del passato, capace di promuovere il consolidamento dell'identità storica italiana ed europea.

Il 3 settembre 2016 il Comune di Predappio e l'Istituto Storico Parri di Bologna sottoscrivono una convenzione con la quale si dà incarico all'Istituto di elaborare un'iniziativa scientifica e museografica nell'ambito del progetto di restauro e ri-funzionalizzazione dell'Ex casa del fascio e dell'ospitalità di Predappio. L'intento è di avviare un processo di ri-memorizzazione del passato che porti alla rimozione nell'immaginario collettivo delle persistenti falsificazioni fasciste:

I centri di documentazione e di esposizione sono l'architrave di tutti i processi di rimemorizzazione, tanto più necessari quanto più il passato è controverso e ha generato memorie divise. [...] L'intento più ambizioso del progetto è quello di consegnare il fascismo alla storia, fondando questo passaggio sul riconoscimento dell'effettiva dimensione storica di un fenomeno che ha segnato profondamente e drammaticamente, come peraltro il comunismo, l'intera storia del XX secolo, ma che si è concluso e che - come tale - non può più tornare.[6]

Nell'ottobre 2016 la Giunta comunale di Predappio approva la costituzione del Comitato Scientifico internazionale, proposto dall'Istituto che assume un ruolo di supporto durante la fase di progettazione dell'esposizione.

Nell'aprile 2017 avviene la consegna al Comune di Predappio del progetto scientifico e museografico intitolato L'Italia totalitaria. Stato e società in epoca fascista. Un'Esposizione permanente. Il progetto è approvato dal Comune e viene accolto positivamente dai rappresentanti istituzionali di Regione Emilia-Romagna, MIBACT e Presidenza Commissione cultura della Camera. A ottobre e dicembre il progetto scientifico e museografico dell'Esposizione permanente è presentato pubblicamente a Roma e a Predappio.

Nei mesi di marzo e ottobre 2018 1'Ex Casa del fascio e dell'ospitalità è aperta al pubblico in collaborazione col FAI. Nel marzo 2019 è sottoscritta una convenzione tra il Comune di Predappio e Ser.In.Ar. Forlì-Cesena per la gestione del centro Progetto Predappio, che procederà all'avvio del piano di ri-funzionalizzazione dell'Ex casa del fascio e dell'ospitalità di Predappio. Il centro prevede un consiglio direttivo presieduto da Giorgio Frassineti, ex sindaco del Comune di Predappio, e composto dai membri Alberto De Bernardi e Gabriele Zelli. La direzione organizzativa è affidata a Carlo Giunchi e quella scientifica a Marcello Flores.

Nel maggio 2019 è conferito l'incarico della progettazione definitiva, della direzione dei lavori e del coordinamento per il restauro e la ri-funzionalizzazione dell'edificio a un raggruppamento temporaneo d'impresa coordinato dallo Studio Valle Progettazioni di Roma.

\section{Un dibattito pubblico: gli storici a confronto}

L'accesa discussione pubblica suscitata dall'iniziativa museografica da realizzarsi a Predappio rappresenta un'occasione significativa di confronto e di riflessione da cui emerge la necessità di attuare nuove forme di ricostruzione e trasmissione della storia del fascismo e di avviare un'attenta riflessione sulle modalità di interazione con il territorio, inteso come elemento regolatore tra passato e presente.

Per molti la criticità risiederebbe soprattutto nella scelta del luogo. Come puntualizza Carlo Ginzburg in un suo intervento pubblicato sulla rivista Doppiozero, il progetto potrebbe portare alla 
realizzazione di un museo celebrativo incentrato sulla figura del duce con il rischio di offrire una visione riduttiva del fascismo basata esclusivamente sul culto mussoliniano:

«Un museo situato a Predappio identificherebbe il fascismo con l'individuo Mussolini, forzando fino alla caricatura il senso dell 'impresa storiografica, discutibilissima, di Renzo de Felice. Perché Predappio, anziché Milano o Roma, città senza le quali la storia del movimento, poi regime fascista, sarebbe impensabile? Ma ̀̀ chiaro: Predappio si presta ai pellegrinaggi, un termine associato al culto.»[7]

Anche Pier Paolo Tamburelli si sofferma sulla scelta del luogo. Riconosce e condivide la necessità espressa dal sindaco di Predappio di fermare l'afflusso di neofascisti attraverso un progetto di riqualificazione territoriale, ma propone di inserirlo in un progetto di ampiezza nazionale per garantire all'iniziativa maggiore scientificità storica e scongiurare il rischio di alimentare sentimenti nostalgici:

«Forse la discussione potrebbe essere più produttiva se si adottassero alcune accortezze che consentirebbero di escludere da subito qualsiasi intento celebrativo. Converrebbe quindi parlare di un centro di documentazione sul fascismo, e vedere poi concretamente all'interno di quale politica culturale sarebbe opportuno inserirlo. Certamente la realizzazione di un centro di documentazione sul fascismo in un piccolo paese della Romagna sarebbe piuttosto curiosa, visto che il fascismo è stato un fenomeno nazionale e merita una riflessione più ampia. Sarebbe quindi necessario che il centro di documentazione di Predappio fosse preceduto da un'istituzione con obiettivi più ampi (e anche molto banalmente più facilmente raggiungibile e più spaziosa di quanto non sia la ex Casa del fascio di Predappio).»[8]

Simon Levis Sullam nel suo intervento sottolinea la natura sacrale di Predappio definendo la città un mnemotopo, cioè un luogo simbolico avulso dalla realtà, che dunque concede al visitatore un'esperienza esclusivamente spirituale:

«Predappio può essere pensato come mnemotopo, in quanto luogo che ha dato origine a un fondatore e in quanto méta di pellegrinaggio presso la tomba del fondatore, in cui persiste una componente religiosa, e dove i pellegrini [...] incontrano la trascendenza. Tutto ciò è molto difficile da azzerare o neutralizzare e ci pone di fronte a un luogo della memoria che ha carattere sacro o sacralizzato.»[9]

Altre criticità sono evidenziate da Enzo Collotti e Filippo Focardi. I due storici esprimono la convinzione che nel nostro Paese non si sia ancora avviata una riflessione storiografica e culturale in grado di promuovere il consolidamento di una visione autocritica e il superamento di stereotipi che hanno portato all'assunzione di un atteggiamento vittimistico nei confronti del passato. In particolare Collotti critica chi si rifà al modello tedesco citando come esempio il Centro di documentazione del nazionalsocialismo di Monaco di Baviera, ospitato nei luoghi dove sorgeva la sede - dal 1920 e al 1933 - del Partito Nazionalsocialista Tedesco dei Lavoratori. Il successo dell'iniziativa tedesca è il risultato, secondo Collotti, di un processo di rielaborazione del passato basato su una precisa politica e cultura della memoria:

«Chi si richiama a iniziative come quella del Museo del Nazional Socialismo inaugurato un anno fa a Monaco di Baviera sottovaluta che alle spalle di questa iniziativa vi sono stati decenni di vive discussioni che hanno riflettuto l'iter della storiografia tedesca sul nazismo e il percorso di una memoria pubblica che ha accettato di fare i conti con il passato, in un processo che peraltro non è mai venuto meno. Anche per noi prescindere da un processo storico politico-culturale di tale portata sarebbe incomprensibile. Proprio per questo l'idea di approfittare dell'occasione 
Predappio per dare luogo ad una iniziativa come quella prospettata appare quantomeno frettolosa e improvvisata.»[10]

Secondo Filippo Focardi occorre superare l'atteggiamento autoassolutorio che ha prevalso nell'opinione pubblica e riconoscere che l'Italia non è stata solo vittima ma anche carnefice. Ciò è possibile solo attraverso un progetto culturale da realizzare in un luogo simbolico per la storia del fascismo:

«Bisogna lavorare sul piano culturale, ormai è prevalso un paradigma valutativo del fascismo che lo ridimensiona, privandolo delle sue caratteristiche repressive, oppressive e criminali che invece ha storicamente avuto. Gli italiani trascurano completamente la dimensione criminale del fascismo e ne hanno un'immagine banale e riduttiva, come di una dittatura all'acqua di rose. [...] Io penso sia necessario un museo importante sul fascismo, ma a Roma che è la città dov'è andato al potere o a Milano dove è nato. A Predappio il museo si presta più alla nostalgia che alla memoria. Fare di Predappio il luogo della visione critica del fascismo è una sfida che può essere persa e non possiamo permettercelo.»[11]

Marcello Flores e Alberto De Bernardi, storici coinvolti direttamente nel progetto, rifiutano la censura preventiva e ribadiscono che soltanto attraverso un'operazione culturale condotta con un approccio rigorosamente scientifico sarà possibile superare tutte le criticità legate alla scelta del luogo. L'obiettivo del progetto, ambizioso ma realizzabile, è quello di illustrare in uno spazio pubblico il racconto di quello che è stato il fascismo, di quello che è stata la società italiana durante il fascismo e quindi di offrire, attraverso un'esposizione museografica, una lezione di storia qualificata e accessibile a tutti.

Il progetto di Predappio ha dato, dunque, avvio a un vivace confronto tra studiosi che, aldilà delle posizioni assunte, riconoscono e condividono l'esigenza di avviare in Italia una riflessione storica più consapevole, capace di cancellare definitivamente nella memoria collettiva una visione mitologica del fascismo e attuare un percorso formativo non soggetto a strumentalizzazioni politiche e ideologiche.

\section{Conclusioni}

Quando Collotti e Focardi denunciano il mancato avvio in Italia di un'operazione storica, politica e culturale che metta in evidenza i crimini del fascismo demistificandone i falsi stereotipi, intendono puntualizzare che il culto di Mussolini non riguarda solo Predappio ma tutto il Paese. Se Predappio è ritenuto un luogo pericoloso, perché slegato dalla realtà storica e vincolato al culto di Mussolini, significa che il ventennio fascista è ancora un ricordo vivo e controverso.

Il sociologo francese Maurice Halbwachs nella sua opera Memoria collettiva spiega che la storia ha inizio quando svanisce la memoria:

Ogni memoria collettiva ha per supporto un gruppo limitato nello spazio e nel tempo. Non si può raccogliere la totalità degli avvenimenti in un unico quadro che a condizione di separarli dalla memoria dei gruppi che ne custodivano il ricordo, di recidere i legami attraverso cui erano uniti alla vita psicologica degli ambienti sociali dove si erano prodotti, e di non conservarne che lo schema cronologico e spaziale. Non si tratta più di rivivere le cose accadute nella loro realtà, ma di ricollocarle nei quadri, esterni ai gruppi, nei quali la storia dispone gli avvenimenti, e di definirli attraverso ciò che li differenzia gli uni dagli altri.[12] 
Esiste dunque una sostanziale differenza tra la memoria collettiva e la storia. Il fattore determinante per la sopravvivenza della prima è la continuità. Soltanto se si mantiene viva nella coscienza del gruppo, la memoria può conservarsi nel tempo. Ma quando il gruppo scompare, essa svanisce. La storia, invece, agisce per precisa selezione e separazione dei fatti dal gruppo di chi ha contribuito a crearli, e procede per classificazione e collocazione nell'ambito di determinate periodizzazioni.

Un'altra differenza fondamentale è che la memoria collettiva ricostruisce il passato in base alle esigenze e alle aspettative del presente, cioè dei membri del gruppo. La storia, invece, agisce rifuggendo i condizionamenti dati dal contesto sociale di appartenenza.

Oltre questa distinzione resta il fatto che in Italia, ancora oggi, il tema del fascismo è oggetto di strumentalizzazioni politiche e culturali che impediscono il consolidarsi di una precisa visione critica nei confronti del proprio passato.

La chiave di lettura per comprendere la genesi di questa mancata autocritica, è da ricercarsi nella contrapposizione dei due stereotipi autoassolutori generati e diffusi in Italia nel dopoguerra: quello del «cattivo tedesco», servo e crudele criminale, e quello del «bravo italiano», vittima di una guerra sventurata e salvatore degli oppressi. Si è preferito, insomma, attribuire alla Germania la responsabilità di tutte le atrocità perpetrate in quegli anni per oscurare le proprie colpe e autoassolversi. Tale atteggiamento è stato, oltretutto, ampiamente condiviso e legittimato sia dalla sinistra antifascista che dalla destra neofascista: occorreva, in entrambi i casi, prendere le distanze dall'ex alleato tedesco e riscattarsi pubblicamente. Negli anni le molte e varie iniziative culturali organizzate per storicizzare il fascismo e i suoi crimini, non sono bastate a superare questa visione distorta e fuorviante.

In questa prospettiva non stupisce, dunque, l'assenza di un consenso unanime al progetto museografico dedicato alla storia del fascismo a Predappio. Forse perché, come ricorda Elena Pirazzoli, «finché si avverte il bruciore della ferita difficilmente si può procedere verso la sanazione definitiva.»[13]

\section{Note:}

[1] I luoghi della memoria. Simboli e miti dell'Italia uniti, Mario Isnenghi (a cura di), Laterza, Roma-Bari, 2010, p. VII

[2]E. Giovannetti, Il caso Predappio, in "IBC" XVIII, 2010, 2(http://rivista.ibc.regione.emiliaromagna.it/xw-201002/xw-201002-d0001/xw-201002-a0023)

[3] Ivi, pp. 570-571

[4] H. Belting, Antropologia delle immagini, Carocci, Roma, 2011, pp.174-175

[5] D. Freedberg, Il potere delle immagini. Il mondo delle figure: reazioni e emozioni del pubblico (1989), Einaudi, Torino, 2009, p. 193

[6] C. Giunchi (Progetto culturale e Programma di valorizzazione) e M. Flores (Coordinamento Progetto Scientifico e Museografico), L'Italia totalitaria. Stato e società in epoca fascista. Un'esposizione permanente. Ex casa del fascio e dell'ospitalità di Predappio, <http://ereview.it/sites/default/images/articles/media/170/12_relazione-progetto-esposizione.pdf> 
[7]C. Ginzburg, Il fascismo non è solo Mussolini, in "Doppiozero", 9 marzo 2016

(http://www.doppiozero.com/materiali/storia-e-memoria/predappio-si-o-no)

[8]P. P. Tamburelli, Documentare il fascismo, in "Doppiozero", 26 marzo 2016, (http://www.doppiozero.com/materiali/storia-e-memoria/una-strategia-generale-sui-centri-didocumentazione-del-fascismo)

[9]S. Levi Sullam, Contro il Museo del fascismo, in Doppiozero, 31 marzo 2016, (http://www.doppiozero.com/materiali/contro-il-museo-del-fascismo-predappio)

[10]E. Collotti, L'inglorioso museo di Predappio, in Il Manifesto, 5 aprile 2016

(https://ilmanifesto.it/cms/wp-content/uploads/2016/02/17/18storie-predappio-bara-mussolini.jpg)

[11]A. Fabozzi, «Più nazisti che fascisti, ma attenti all'allarmismo strumentale». Intervista a Filippo Focardi, 10 dicembre 2017, (https://ilmanifesto.it/piu-nazisti-che-fascisti-ma-attentiallallarmismo-strumentale/)

[12] M. Halbwachs, La memoria collettiva (1968), Nuova edizione critica, P. Jedlowski e T. Grande (a cura di), Unicopli, Milano, 2001, p. 161

[13] E. Pirazzoli, A partire da ciò che resta. Forme memoriali dal 1945 alle macerie del Muro di Berlino, Diabasis, Reggio Emilia, 2010, p. 54 